\title{
Comment on The human sex odds at birth after the atmospheric atomic bomb tests, after Chernobyl, and in the vicinity of nuclear facilities, Hagen Scherb \& Kristina Voigt Environ, Sci Pollut Res (2011) 18:697-707
}

\author{
François Bochud • Thomas Jung
}

Received: 13 October 2011 / Accepted: 16 January 2012 / Published online: 11 February 2012

(C) Springer-Verlag 2012

Scherb and Voigt (2011a) examine the sex ratio, also called sex odds, defined as the ratio of live births of boys and girls in the period 1950 to 2007 . They observe its annual values for different regions and countries and examine their year-to-year variation with regard to two major radiological events: the Partial Nuclear Test Ban Treaty (PNTBT) and the Chernobyl accident. It also presents results of a non-peer-reviewed study (Kusmierz et al. 2010) on the sex ratio at different distances from Swiss and German nuclear power plants (NPPs). The main conclusion Scherb and Voigt draw from their results is that the international consensus on the health effects of ionizing radiations is underestimated by several orders of magnitude. The authors also wrote the editorial in the same issue of the journal (Scherb and Voigt 2011b) and took this point further by announcing that there are "one million missing children across Europe and parts of Asia after Chernobyl till to date."

These extraordinary claims, if true, would require a drastic change in our use of ionizing radiations. Such claims cannot be accepted unless they are backed by extraordinary evidence. The purpose of this letter is not to counter the potential consequences of these assertions but to set out the

Responsible editor: Philippe Garrigues

F. Bochud $(\bowtie)$

Institute of Radiation Physics,

Lausanne University Hospital,

Lausanne, Switzerland

e-mail: Francois.Bochud@chuv.ch

T. Jung

Div. Effects and Risks of Ionizing and Non-Ionizing Radiation,

Dept. Radiation Protection and Health,

Federal Office for Radiation Protection,

Oberschleissheim, Germany reasons why we are not convinced by the arguments put forward by Scherb and Voigt.

Scherb and Voigt begin with the temporal variation of the sex ratios in Europe and the USA in two different figures (one around the time of the ratification of the PNTBT and one around 1986). It would have been fairer to plot them together in one figure (see Fig. 1). In the period in which European data overlap (23 and 39 countries were taken into account, respectively), the difference between the two sets of data is typically about 0.002 . Globally, the spread of the data points along the authors' fits is 0.005 between the highest and the lowest values, and along the whole period of time considered by the authors, the European values fluctuate between a minimum of 1.055 and maximum of 1.065. Therefore, we would have expected that the authors state that (1) there are some uncertainties, (2) a rough estimate is about 0.005 , and (3) they did not perform such an analysis. In particular, we could have expected to see a comparison with a stable behavior of the sex ratio as well as an increase at any year. A much lower $p$ value for a specific increase in year 1963 or 1986 would have been more compelling than a nice-looking fit performed only in these two specific years. For the American data, the increase identified by the authors during the 1960-1975 period should have been put in relation with another study that showed that this was mainly attributable to the African-American population and is not yet understood (Davis et al. 2007).

Based on their assumption that an effect on the European sex ratio has been shown in 1987, the Scherb and Voigt paper presents the Russian sex ratio between 1980 and 2000 , where an increase is clearly visible. The authors attribute this observation solely to the year 1986, and the curve is fitted by a step function. No statistical test is performed for any other year, and no regional analysis is performed to search for a potential difference between the 
Fig. 1 European and American sex ratios from 1950 to 2007 (Figures 1 and 2 of Scherb and Voigt combined)

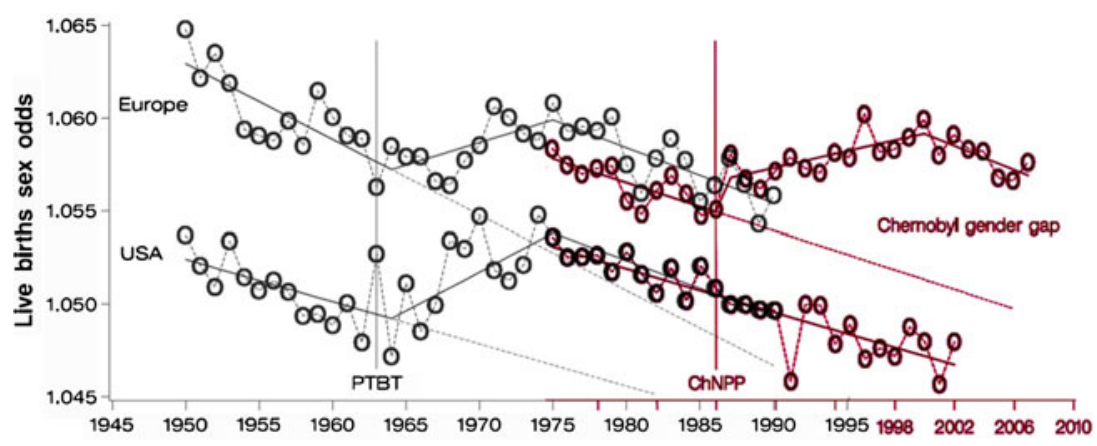

18 most affected regions that received an average effective dose from the Chernobyl accident higher than $0.7 \mathrm{mSv}$ during the 1986-2005 period (population about 30 million) and the rest of the country (population about 117 million) (UNSCEAR 2011). In support of their claim that there is a causal relationship between the dose and the observed variations of the sex ratio in Russia, the authors show that the increase in the German data is smaller and that an even smaller increase is seen in the French data in 1986. Figure 2 has been plotted with the data recommended by the authors $\left(\mathrm{HFADB}^{1}\right.$ ) for Russia, Italy, and France. We chose to show the data for Italy, because its population is comparable to France and Germany, but it received a higher mean effective dose than Germany (UNSCEAR 2011, page 141). We recommend the reader hide the horizontal scale and try to define where a step function should be placed in order to fit the data. Once this qualitative exercise is performed, any skeptical reader would expect to see the results of the fits performed for an increase at each year of the considered period and not only in 1987.

The hypothesis that a general, slow increase of sex ratios was occurring in Russia during the 1980-2000 period has not been tested by the authors. This is unfortunate because sex ratio increases have been observed in many Asian countries throughout this period (Zhu et al. 2009). The main reason for this increase is that selective-gender abortions have become common since prenatal ultrasound examinations became widely available in the 1980s and have led to sex ratios up to 1.15 or more (Hesketh and Zhu 2006). We understand that this social behavior seems not to have been reported in Russia. However, this potential simple explanation would have necessitated a much lower amplitude than the one observed in neighboring countries.

Scherb and Voigt then assume that a cause-effect relationship was established and so search for deficits in the number of births in 34 European countries in the year following the Chernobyl accident. However, the only country they find a deficit in is Denmark. They look at the absolute number of live births in the time period 1984-1990, in which an increasing

\footnotetext{
${ }^{1}$ Health For All Data Base managed within the WHO framework http://data.euro.who.int/hfadb
}

trend can be seen. The authors conclude that a deviation from this trend is observable for the year 1987. In the results section of their paper, the authors rightly caution about the significance of this observable departure from a straight line. However, further down in the text, they use this observation and another of their previous publications in order to extrapolate it to the whole of Europe and the USA and to estimate a "gender gap" of two million people, which they then use as a proof that the risk estimate of low-dose ionizing radiation has been greatly underestimated by the international scientific community. Figure 3 presents the same data but over a longer time scale. The "trend" observed by the authors (and shown as a continuously increasing line) now completely disappears. With the approach of picking up only the data that confirm their prior beliefs, the authors were almost certain to find an increase in 1987. By discarding 33 countries, the authors kept only $3 \%$ of the data in order to show an effect. Basic statistical courses usually teach students that we should leave less than $5 \%$ chance to the null hypothesis before starting to think that an effect is statistically significant. Interestingly, Scherb and Voigt propose to do the exact opposite.

As further evidence of a possible relationship between radiation and sex ratio, Scherb and Voigt then turn their attention to the distance between inhabited locations and German and Swiss NPPs. It is difficult to fully assess their results, because the methodology used was not published in a peer-reviewed study (Kusmierz et al. 2010), no confounding factors were taken into account, and no alternative explanations were tested. The fact that the study identified a sex ratio peak at $14.3 \mathrm{~km}$ and not within 0 to $5 \mathrm{~km}$ from the NPP is difficult to understand in a cause-effect framework. Another shortcoming in their reasoning is the absence of a documented relationship between the distance to the NPP and the dose delivered to the inhabitants. Finally, the last section of the Kusmierz et al. paper (2010) demonstrates the author's own doubts when they mention that "[e]xtended investigations are required to support or refute the findings of this paper." We think that these doubts should have been quoted in the Scherb and Voigt paper in order to help the reader to better understand the speculative nature of the study they are referring to.

Before concluding this letter, we would like to mention two fundamental biological aspects that are important for 
Fig. 2 Sex ratios of Russia, Italy, and France (HFADB database). German data are not plotted on this graph as they were only available for the period since 1990

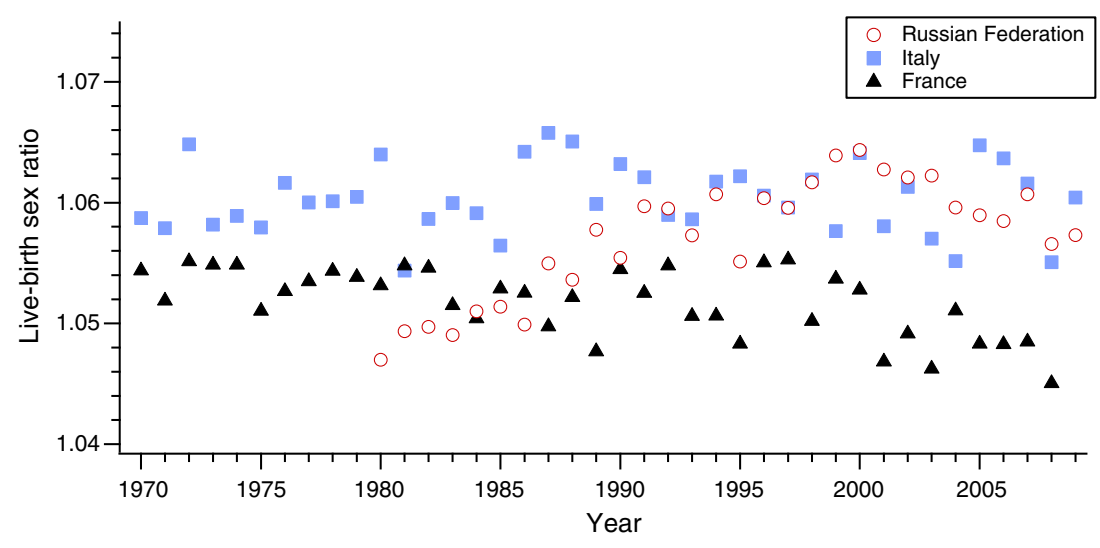

understanding the sex ratio variations described by Scherb and Voigt: (1) the sex ratio varies significantly in the period between conception and birth and (2) the effect of radiations on the sex ratio has been addressed in the literature.

The sex of a human being is primarily determined at the time of conception and depends on whether the fertilizing sperm bears an $\mathrm{X}$ or a $\mathrm{Y}$ chromosome. As $\mathrm{X}$ - and Y-bearing sperms are produced in equal numbers during spermatogenesis, theoretically, a sex ratio at conception (primary sex ratio) close to unity should be expected. In humans, it has been found that the primary sex ratio or the sex ratio in the first days of embryogenesis differ remarkably from this theoretically expected ratio of $1: 1$, and may be as high as 170 males to 100 females (Hassold et al. 1983; Pergament et al. 2002). Because the secondary sex ratio (i.e., at birth) is in the range of 1.04 to 1.07 , it is obvious that pre- and postimplantation embryonic as well as prenatal fetal mortality affects male and female embryos and fetuses differently. During gestation, a continuous decline of the sex ratio has been observed in mammals in general (Jongbloet 2004) as well as in humans (Kukharenko 1970). However, it should be noticed that fewer than $25 \%$ of natural human fertilizations survive to term, even with healthy parents (Boklage 2005). The highest embryonic loss, in the range of two thirds of fertilized oocytes, takes place between conception and the time of implantation or immediately thereafter, i.e., before clinical recognition of the pregnancy. Major sex ratio determining factors are operating at or around the time of conception among which the hormonal status of the mother (James 2011) and maternal diet (Grant and Chamley 2010) seem to be significant ones. Later during pregnancy, natural selection is further putting pressure mainly on less viable male fetuses. This mechanism was extensively described by Trivers and Willard (1973) and more recently reviewed by Wells (2000). In special circumstances, this mechanism may select against female fetuses, however, on a much lower level (Catalano et al. 2009). The "gender gap" claimed by Scherb and Voigt can therefore also be interpreted as a reduction in male embryonic loss during embryogenesis instead of a reduction of female births.

In their editorial, Scherb and Voigt (2011b) refer to the publication of Schull and Neel (1958) in which, based on preliminary data, an effect of ionizing radiation on sex ratio was described. However, the same authors reported some years later (Schull et al. 1966) that based on an extended data set and a longer follow-up, their earlier preliminary results were not confirmed. This later publication is ignored by the authors. Furthermore, two more recent studies (Winther et al. 2003; Reulen et al. 2007) showed that the sex ratio among the children of parents treated by radiation therapy was not significantly different to the rest of the population. Therefore, when Scherb and Voigt contend that this phenomenon has not been investigated at all by national or international
Fig. 3 Absolute live births and sex ratios observed in Denmark (HFADB database). The "selected time period" was chosen by the authors of the study in order to derive a trend. A high value of the sex ratio is observed in 1971, 1976, and 1987

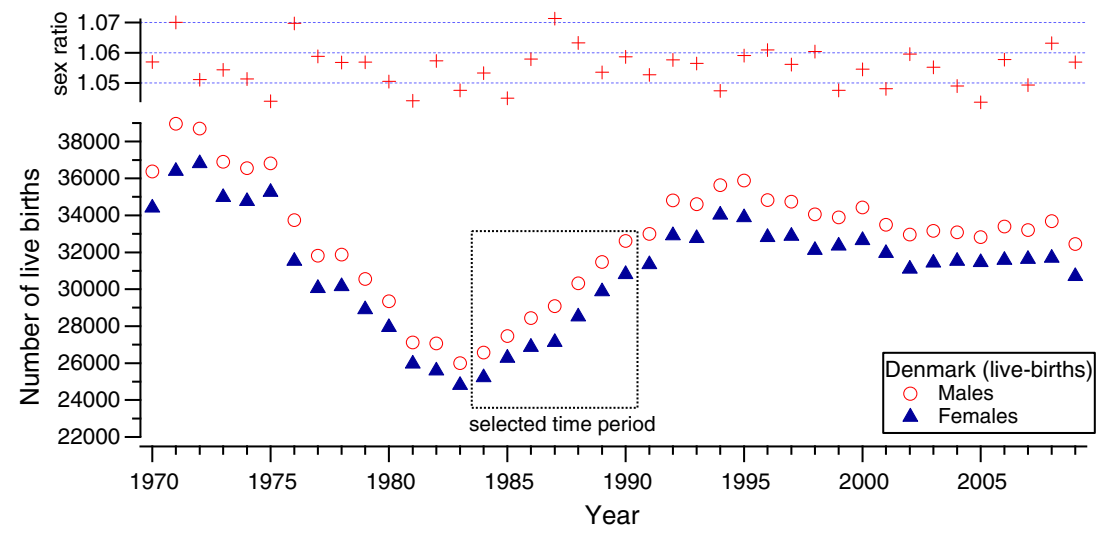


institutions, nor by the scientific community, their criticism is an empty one. From empirical data on exposed human populations, there is to date no established effect of ionizing radiation on the sex ratio of newborn infants.

\section{Conclusion}

The sex ratio could be affected by ionizing radiation, but the potential confounding factors are so numerous that its use as an indicator for radiation-induced health effects is not likely to be of any value. We fully admit the value and the power of sound epidemiological studies. However, understanding the biological effects of ionizing radiations will continue to require the rigorous approach of radiobiology, which has the advantage of providing explanations and not just correlations.

Even if we put this aspect aside, the arguments provided by Scherb and Voigt are not convincing and do not provide reasons of concern for public health. Most of the trends identified by their study disappear when larger periods of time are considered, and their dose-effect estimation is based on one observation in one country not reproduced in any of the other 33 European countries. The only clear and progressive increase of sex ratios shown in this study is that in Russia between 1980 and 2000. However, Scherb and Voigt do not provide any scientific proof of a stepwise increase in 1987, and they did not attempt to compare this with similar trends observed in the same period of time in many Asian countries albeit with much higher amplitude.

Using this scant evidence, Scherb and Voigt go far beyond a sole effect on the sex ratio at birth. They make the extraordinary claim that "the internationally established radiation risk concept based on average absorbed dose is in error at three to four orders of magnitude or, more likely, it is conceptually wrong" and that there are at least "one million missing children." If this were true, there would be dramatic consequences. For example, the use of ionizing radiations in diagnostic and therapeutic medicine would need to be immediately examined and probably drastically reduced. If the authors' claim was correct, many effects should already be observable in patients. Therefore, continuing the generally accepted practice of applying the precautionary principle and radiation protection policies recommended by the ICRP is still reasonable and coherent with the current state of good science.

\section{References}

Boklage CE (2005) The epigenetic environment: secondary sex ratio depends on differential survival in embryogenesis. Hum Reprod 20(3):583-587
Catalano RA, Saxton K, Bruckner T, Goldman S, Anderson E (2009) A sex-specific test of selection in utero. J Theor Biol 257(3):475479

Davis DL, Webster P, Stainthorpe H, Chilton J, Jones L, Doi R (2007) Declines in sex ratio at birth and fetal deaths in Japan, and in U.S. whites but not African Americans. Environ Health Perspect 115 (6):941-946

Grant VJ, Chamley LW (2010) Can mammalian mothers influence the sex of their offspring peri-conceptionally? Reproduction 140:425433

Hassold T, Quillen SD, Yamane JA (1983) Sex ratio in spontaneous abortions. Ann Hum Genet 47(Pt 1):39-47

Hesketh T, Zhu WX (2006) Abnormal sex ratios in human populations: causes and consequences. PNAS 103(36):13271-13275. doi: $10.1073 /$ pnas.0602203103

James WH (2011) The categories of evidence relating to the hypothesis that mammalian sex ratios at birth are causally related to the hormone concentrations of both parents around the time of conception. J Biosoc Sci 43:167-184

Jongbloet PH (2004) Over-ripeness ovopathy: a challenging hypothesis for sex ratio modulation. Hum Reprod 19(4):769-774. doi:10.1093/humrep/deh136

Kukharenko VI (1970) The primary sex ratio in man (analysis of 1014 embryos). Genetika 6(5):142-149

Kusmierz R., Voigt K., Scherb H (2010) Is the human sex-odds at birth distorted in the vicinity of nuclear facilities (NF)? A preliminary geo-spatial-temporal approach. In: Greve K, Cremers AB (eds) 24th EnviroInfo 2010. Bonn and Cologne, Germany, October 6th-8th 2010. Shaker, pp 616-626

Pergament E, Toydemir PB, Fiddler M (2002) Sex ratio: a biological perspective of 'Sex and the City'. Reprod Biomed Online 5 (1):43-46

Reulen RC, Zeegers MP, Lancashire ER, Winter DL and Hawkins MM, on behalf of the British Childhood Cancer Survivor Study (2007) Offspring sex ratio and gonadal irradiation in the British Childhood Cancer Survivor Study. Brit J Cancer 96:1439-1441

Scherb H, Voigt K (2011a) The human sex-odds at birth after the atmospheric atomic bomb tests, after Chernobyl, and in the vicinity of nuclear facilities. Environ Sci Pollut Res 18:697-707. doi:10.1007/s11356-011-0462-z

Scherb H, Voigt K (2011b) Adverse genetic effects induced by chemical or physical environmental pollution. Environ Sci Pollut Res 18:695-696. doi:10.1007/s11356-010-0332-0

Schull WJ, Neel JV (1958) Radiation and the sex ratio in man. Science 128(3320):343-348

Schull WJ, Neel JV, Hashizume A (1966) Some further observations on the sex ratio among infants born to survivors of the atomic bombings of Hiroshima and Nagasaki. Am J Hum Genet 18 (4):328-338

Trivers RL, Willard DE (1973) Natural selection of parental ability to vary the sex ratio of offspring. Science 179(68):90-92

UNSCEAR (2011) Sources and effects of ionizing radiation. UNSEAR 2008 report to the General Assembly with scientific annexes, Volume II, Annexes C, D and E. United Nations, New-York.

Zhu WX, Lu L, Hesketh T (2009) China's excess males, sex selective abortion, and one child policy: analysis of data from 2005 national intercensus survey. Br Med J 338:b1211. doi:10.1136/bmj. b1211

Wells JCK (2000) Natural selection and sex differences in morbidity and mortality in early life. J Theor Biol 202:65-76

Winther JF, Boice JD Jr, Thomsen BL, Schull WJ, Stovall M, Olsen JH (2003) Sex ratio among offspring of childhood cancer survivors treated with radiotherapy. Br J Cancer 88:382-387 